\title{
Comparaison des effets de la contrainte hydrique sur la croissance, la conductance stomatique et la photosynthèse de jeunes plants de chênes méditerranéens (Quercus suber, $Q$. faginea, Q. coccifera) en Tunisie
}

\author{
Mustapha Ksontini ${ }^{\mathrm{a}}$, Philippe Louguet ${ }^{\mathrm{b}}$, Daniel Laffray ${ }^{\mathrm{b}}$, \\ Mohamed Nejib Rejeb ${ }^{a}$
}

\footnotetext{
${ }^{a}$ Institut national de recherche en génie rural, eaux et forêts, BP 2, 2080 Ariana, Tunisie

${ }^{b}$ Laboratoire de physiologie végétale, Université Paris Val-de-Marne, Avenue du Général-de-Gaulle 94010 Créteil, France
}

(Reçu le 12 septembre 1996 ; révisé le 9 janvier 1997 ; accepté le 17 septembre 1997)

\begin{abstract}
Résumé - Une étude comparative des réponses physiologiques de jeunes plants de chênes sempervirents (chêne liège : Quercus suber $\mathrm{L}$. et chêne kermès : $Q$. coccifera $\mathrm{L}$.) et d'un chêne caducifolié (chêne zéen : $Q$. faginea Willd) a été menée en pépinière à Tunis. Des plants de 6 et 18 mois, élevés en pots, ont été soumis à une contrainte hydrique par arrêt d'arrosage pendant deux cycles de dessèchement consécutifs d'une durée de deux mois. Nous avons mesuré les potentiels hydriques de base et minimum, la perte en eau par rapport à la capacité au champ (transpiration cumulée des plantes), la conductance stomatique et l'assimilation photosynthétique. La biomasse des tiges, des racines, les rapports biomasse tige/racine et la surface foliaire ont été également déterminés. Le rapport biomasse tige/racine a diminué davantage au cours de la contrainte hydrique chez $Q$. faginea que chez $Q$. coccifera. La diminution de la conductance stomatique, liée à la chute du potentiel hydrique foliaire de base, était plus précoce chez $Q$. faginea. Les stomates de $Q$. coccifera sont restés encore partiellement ouverts pour des potentiels de l'ordre de $-3 \mathrm{MPa}$. Les réponses de $Q$. suber étaient intermédiaires. La photosynthèse nette et la conductance stomatique étaient étroitement corrélées et présentaient toutes deux une dépression de milieu de journée. Nos résultats indiquent qu'il serait nécessaire d'approfondir les études écophysiologiques en fonction de l'âge des plants et des feuilles au stade juvénile, pour mieux comprendre le comportement des chênes, notamment ses difficultés de régénération spontanée. (C) Inra/Elsevier, Paris.)
\end{abstract}

Conductance stomatique / photosynthèse / sécheresse / Quercus suber / Quercus coccifera / Quercus faginea

\footnotetext{
* Correspondance et tirés à part

E-mail : laffray@univ.paris.fr
} 


\begin{abstract}
Comparison of the water stress effects on stomatal conductance, photosynthesis and growth of Mediterannean oak seedlings (Quercus suber L., $Q$. faginea, $Q$. coccifera) in Tunisia. Two evergreen oaks (Quercus suber $\mathrm{L}$., $Q$. coccifera) and one deciduous oak $(Q$. faginea Willd.) were subjected to drought in a nursery in Tunis. Six- and 18-month-old seedlings were grown in pots and underwent two cycles of drought by withholding water supply. Predawn and midday leaf water potentials, stomatal conductance, photosynthesis and plant transpiration were recorded. An analysis of root and shoot biomass, shoot-to-root ratio and leaf area was also performed. Our results showed a higher reduction of shoot-to-root biomass ratio of $Q$. faginea compared to $Q$. coccifera under water limitation. The stomatal conductance decrease, related to the decrease of predawn leaf water potential, occurred earlier with $Q$. faginea. $Q$. coccifera kept its stomata partly open at a water potential of $-3.0 \mathrm{MPa}$. Q. suber behaved somewhat intermediate. Photosynthesis and stomatal conductance were correlated and showed a midday depression. Our results indicate that more ecophysiological studies are required to take into account seedling and leaf ages during the juvenile stage for a better understanding of the water stress responses of these species and regeneration problems of oaks. (@ Inra/Elsevier, Paris.)
\end{abstract}

Stomatal conductance / photosynthesis / drought / Quercus suber / Quercus coccifera / Quercus faginea

\section{INTRODUCTION}

La forêt et les espaces naturels méditerranéens sont des écosystèmes fragiles, soumis à des perturbations diverses liées étroitement aux conditions du milieu physique et humain. Les risques d'un déséquilibre, d'une dégradation ou d'un dépérissement sont réels, dûs aux effets de la sécheresse en particulier $[9,13]$. Emberger [16] a souligné que l'ampleur de la sécheresse peut expliquer la distribution des différentes espèces de Quercus dans l'aire isoclimatique méditerranéenne.

Les facteurs essentiels qui font échec à la reconstitution naturelle par semis des chênaies sont les facteurs anthropiques et de prédation ainsi que l'insuffisance de la gestion forestière, les facteurs climatiques (sécheresse et chaleur estivale) ainsi que la couverture par le maquis et la strate arbustive $[4,19,23,25]$.

L'adaptation morphologique racinaire de $Q$. coccifera et son anatomie foliaire lui permettent d'extraire l'eau disponible $[28,38]$. Salleo et Lo Gullo [33] ont démontré que la structure des feuilles de $Q$. ilex et $Q$. suber intervient très peu dans l'économie d'eau.

Pour Q. coccifera, la fermeture de midi se produit moins souvent et l'assimilation nette du $\mathrm{CO}_{2}$ est plus élevée que chez les autres espèces. $Q$. coccifera présente beaucoup moins d'uniformité dans la distribution des ouvertures des stomates que $Q$. suber et Arbutus unedo; ces différences sont peut être à mettre en relation avec le fait que cette espèce est plus adaptée aux conditions d'un été aride $[7,39]$.

Acherar et Rambal [2] ont montré l'existence d'une forte corrélation entre le potentiel hydrique de base et la conductance stomatique (gs) maximale chez les chênes sempervirents $(Q$. ilex, $Q$. suber $)$ et chez les caducifoliés ( $Q$. afares et $Q$. faginea).

Les gs maximales mesurées chez $Q$. suber sur des jeunes plants et des plants âgés de 2 ans sont de l'ordre de 0,115 et $0,28 \mathrm{~mol} \mathrm{~m}^{-2} \mathrm{~s}^{-1}$ respectivement $[2,42]$; chez $Q$. coccifera avec des arbres adultes, elles valent de 0,33 à $0,39 \mathrm{~mol} \mathrm{~m}^{-2} \mathrm{~s}^{-1}$ $[30,40]$, et chez $Q$. faginea elles atteignent $0,53 \mathrm{~mol} \mathrm{~m}^{-2} \mathrm{~s}^{-1}$ avec des plants âgés de 2 ans [2].

La dépression de midi de gs est trouvée chez de nombreuses espèces notamment méditerranéennes et permet de limiter l'évaporation au moment où le déficit de saturation en vapeur d'eau de l'air (VPD) est maximal [35]. Il a été noté aussi que les chênes sempervirents peuvent réaliser 
la photosynthèse à un potentiel hydrique très négatif tandis que les caducifoliés évitent la contrainte hydrique par une fermeture plus précoce des stomates [39].

Les populations de chênes liège sont en régression et plus généralement les chênaies méditerranéennes [26]. Des dépérissements des chênes ont été signalés en France comme celui de Quercus robur $[6,14,24]$ et de Quercus cerris en Italie [41]. L'apparition de dépérissements dans les massifs européens ou Nord américains est fréquemment corrélée à des épisodes de sécheresse plus ou moins intenses $[8,9,13]$.

Parmi les causes possibles de l'absence de régénération du chêne liège dans les forêts tunisiennes, nous nous sommes particulièrement intéressés aux effets de la contrainte hydrique.

Davies [12] a souligné l'importance d'étudier les différences d'adaptation à la sécheresse aux stades juvéniles parcequ'il existe un taux élevé de mortalité des jeunes après transplantation pour les espèces ligneuses méditerranéennes.

L'objectif de cette étude est de comparer le comportement écophysiologique du chêne liège $(Q$. suber) avec celui du chêne zéen ( $Q$. faginea, espèce plus sensible), et du chêne kermès $(Q$. coccifera) plus résistant à la contrainte hydrique, en utilisant des jeunes plants élevés dans des pots et d'âges différents. Cette étude s'est poursuivie pendant deux cycles de dessèchement consécutifs séparés par une phase de réhydratation.

Ce travail a pour objectif principal de détecter des traits biologiques de tolérance à la sécheresse susceptibles d'être retenus pour l'amélioration de la résistance à la sécheresse, marqueurs qui peuvent être morphologiques et/ou physiologiques. Les expérimentations menées en conditions naturelles avec contrôle de la contrainte hydrique appliquée à des plants jeunes par arrêt d'arrosage, ont permis d'évaluer les effets du déficit hydrique à plusieurs niveaux : potentiel hydrique, conductance stomatique, photosynthèse, croissances racinaire et caulinaire et surface foliaire.

\section{MATÉRIELS ET MÉTHODES}

Cette expérimentation a été conduite dans la pépinière de l'Institut national de recherches en génie rural eaux et forêts à Tunis en 1995. Les glands sont issus de trois sites différents: Chêne liège de Bellife et Chêne kermès de Ras Rajel en bioclimat subhumide et Chêne zéen de Mtir en bioclimat humide (région du NordOuest tunisien). Les semis ont été effectués à deux périodes : au début des mois de Février 1994 (plants âgés) et janvier 1995 (plants jeunes) dans des seaux de $31 \cong 4 \mathrm{~kg}$ de terreau ( $1 / 3$ sable $+2 / 3$ humus provenant des suberaies). Sa texture est sablono-argileuse ( $73 \%$ de sables fin et grossier et $24 \%$ d'argile et limon); sa composition minérale est de $4 \% \mathrm{P}_{2} \mathrm{O}_{5}$, $3,3 \%$ de matière organique et un $\mathrm{pH}=7,5$. L'humidité pondérale du sol à la capacité au champ est de l'ordre de $26 \%$.

Les plants ont été arrosés régulièrement jusqu'au 9 mai 1995 pour les plants âgés et jusqu'au 26 juin 1995 pour les plants jeunes. Par la suite, deux régimes hydriques ont été appliqués : les plants témoins ont été bien irrigués et les plants stressés ont été soumis à une contrainte hydrique par arrêt total d'arrosage. La partie supérieure des seaux a été protégée par un couvercle muni d'un trou pour le passage de la tige afin de limiter l'évaporation du sol. Deux cycles de dessèchement de 21 et $22 \mathrm{j}$ pour les plants jeunes et 28 et 29 j pour les plants âgés, séparés par une phase de réhydratation de $3 \mathrm{j}$ à la capacité au champ, ont été appliqués.

La conductance stomatique, le rayonnement photosynthétiquement actif (PAR vertical incident), les températures foliaires et de l'air et l'humidité relative de l'air ont été mesurés à l'aide d'un poromètre $\Delta \mathrm{T}$ type AP4 tous les trois à cinq jours à 9 heures du matin et à midi jusqu'à la fin du $1^{\text {er }}$ cycle où la fermeture complète des stomates s'est produite après $21 \mathrm{j}$ (pour les plants jeunes) et $28 \mathrm{j}$ (pour les plants âgés). Ces mesures ont été effectuées sur les mêmes feuilles de rang 3 à 6 (feuilles jeunes pour les plants jeunes et feuilles de l'année pour les plants âgés) sur dix sujets témoins et dix stressés des plants de chêne liège âgés, sur cinq témoins et cinq stressés des plants de chêne liège jeune et chêne kermès âgé, et sur 
trois témoins et trois stressés des plants de chêne kermès jeune et chêne zéen jeune.

Simultanément, deux individus de chaque espèce provenant des plants stressés ont été prélevés pour la mesure de leur potentiel hydrique de base et de midi à l'aide d'une chambre à pression (soil moisture equipment model 3005).

Les dix pots des échantillons stressés de chaque espèce ont été pesés périodiquement (tous les 3 à $5 \mathrm{j}$ au début du cycle de dessèchement et tous les deux jours à la fin du cycle) pour mesurer l'évolution de la perte en eau du sol exprimée en pourcentage de la capacité au champ.

L'assimilation photosynthétique (A) a pu être mesurée seulement pendant le $1^{\text {er }}$ cycle à 9 heures et à midi sur des plants âgés à l'aide du système de mesure de photosynthèse ADC (leaf chamber analysis system type LCA-3). À la fin de chaque cycle de dessèchement, des échantillons de chêne liège âgé (dix témoins et dix stressés), de chêne kermès âgé et de chêne liège jeune (cinq témoins et cinq stressés), de chêne kermès jeune et de chêne zéen jeune (trois témoins et trois stressés), ont été prélevés pour permettre l'analyse des caractéristiques morphologiques : biomasse des tiges, racines et rapports tiges/racines $(T / R)$ après séchage dans une étuve à $80^{\circ} \mathrm{C}$ et pendant $48 \mathrm{~h}$. Les surfaces foliaires (SF) ont été mesurées après détermination de la masse surfacique moyenne des feuilles et du poids sec total de toutes les feuilles par plant. La surface foliaire est obtenue à partir de silhouettes des feuilles sur papier de masse surfacique connue.

Les racines ont été prélevées après élimination du sol par trempage dans l'eau.

L'analyse statistique des données a porté sur les facteurs morphologiques et la conductance stomatique ainsi que sur les données climatiques pendant l'expérimentation.

Pour l'analyse de variance et l'ajustement polynomial, nous avons fait appel à la procédure GLM (general linear models) du SAS et pour la classification multiple des moyennes, nous avons utilisé le test de Duncan.

\section{RÉSULTATS}

\subsection{Les facteurs de l'environnement}

Les analyses statistiques par la procédure GLM (general linear models) du SAS et l'analyse globale des moyennes par le test des variables multiples de Duncan ont mis en évidence une différence hautement significative au seuil de 0,01 pour la $\mathrm{T}^{\circ} \mathrm{C}$, l'HR et le PAR, pour les deux périodes d'expérimentation (période des âgés : 9 mai au 4 juillet 1995 et période des jeunes : 26 juin au 8 août 1995) et entre les deux moments de mesure journalière ( 9 heures et 12 heures).

Nous avons mesuré des PAR et des $\mathrm{T}{ }^{\circ} \mathrm{C}$ plus élevées pour la période d'étude des jeunes plants que pour les âgés avec 725 et $550 \mu \mathrm{mol} \mathrm{m} \mathrm{m}^{-2} \mathrm{~s}^{-1}$ et 35 et $30,5^{\circ} \mathrm{C}$ respectivement. La moyenne des $\mathrm{HR}$ est de 30 et $41 \%$ pour les jeunes et âgés respectivement.

La comparaison entre les données prises le matin et à midi montre que les valeurs des PAR et des $T^{\circ}$ sont plus élevées à 12 heures qu'à 9 heures avec 754 et $431 \mu \mathrm{mol} \mathrm{m} \mathrm{m}^{-2}$, et $33{ }^{\circ} \mathrm{C}$ et $31^{\circ} \mathrm{C}$ respectivement.

Il en ressort que les conditions climatiques des deux périodes d'expérimentations diffèrent significativement entre les deux cycles successifs et entre les expérimentations sur les plants jeunes et âgés. En conséquence, il sera difficile d'évaluer un éventuel effet de l'âge sur les réponses biologiques.

\section{2. Évolution des potentiels hydriques en fonction du temps}

Les variations des potentiels hydriques de base et de milieu de journée pendant les deux cycles de sécheresse sont présentées pour les plants de chêne kermès Ras Rajel âgés (CK), (figure laa) et jeunes (figure lac) et pour les plants jeunes de chêne liège Bellif (CL) (figure Iba) et de chêne zéen (CZ) (figure lbc).

Au début du $1^{\mathrm{er}}$ cycle de dessèchement, les potentiels hydriques de base étaient presque identiques pour toutes les espèces et variaient entre $-0,35$ et $-0,45 \mathrm{MPa}$. Le potentiel hydrique à mi-journée montre 


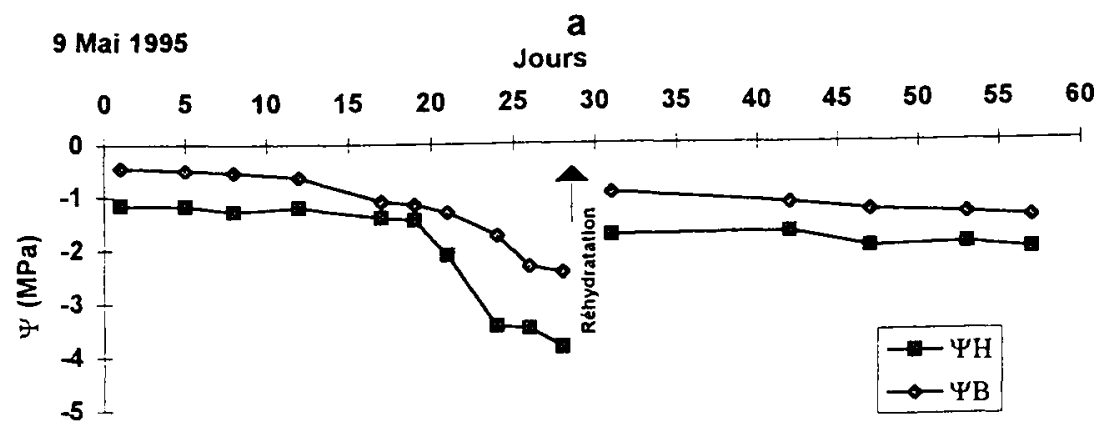

b
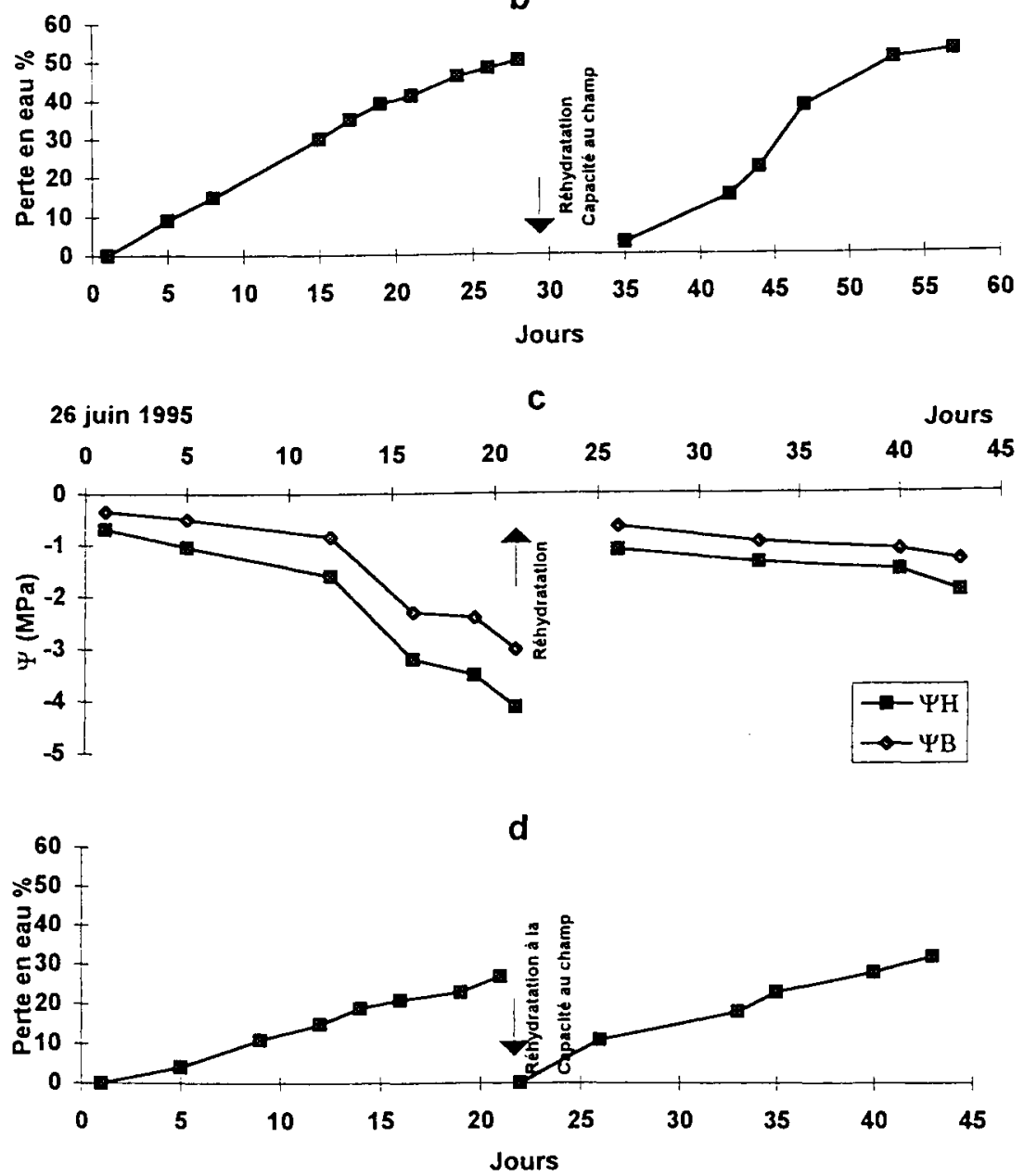

Figure 1a. Variations du potentiel hydrique de base $(\Psi B)$, du potentiel de milieu de journée $\left(\Psi^{\top} \mathrm{H}\right)$, de la perte en eau par rapport à la capacité au champ (Pe) de plants âgés (a et b) et jeunes (c et d) de chêne kermès pendant deux cycles de sécheresse. 

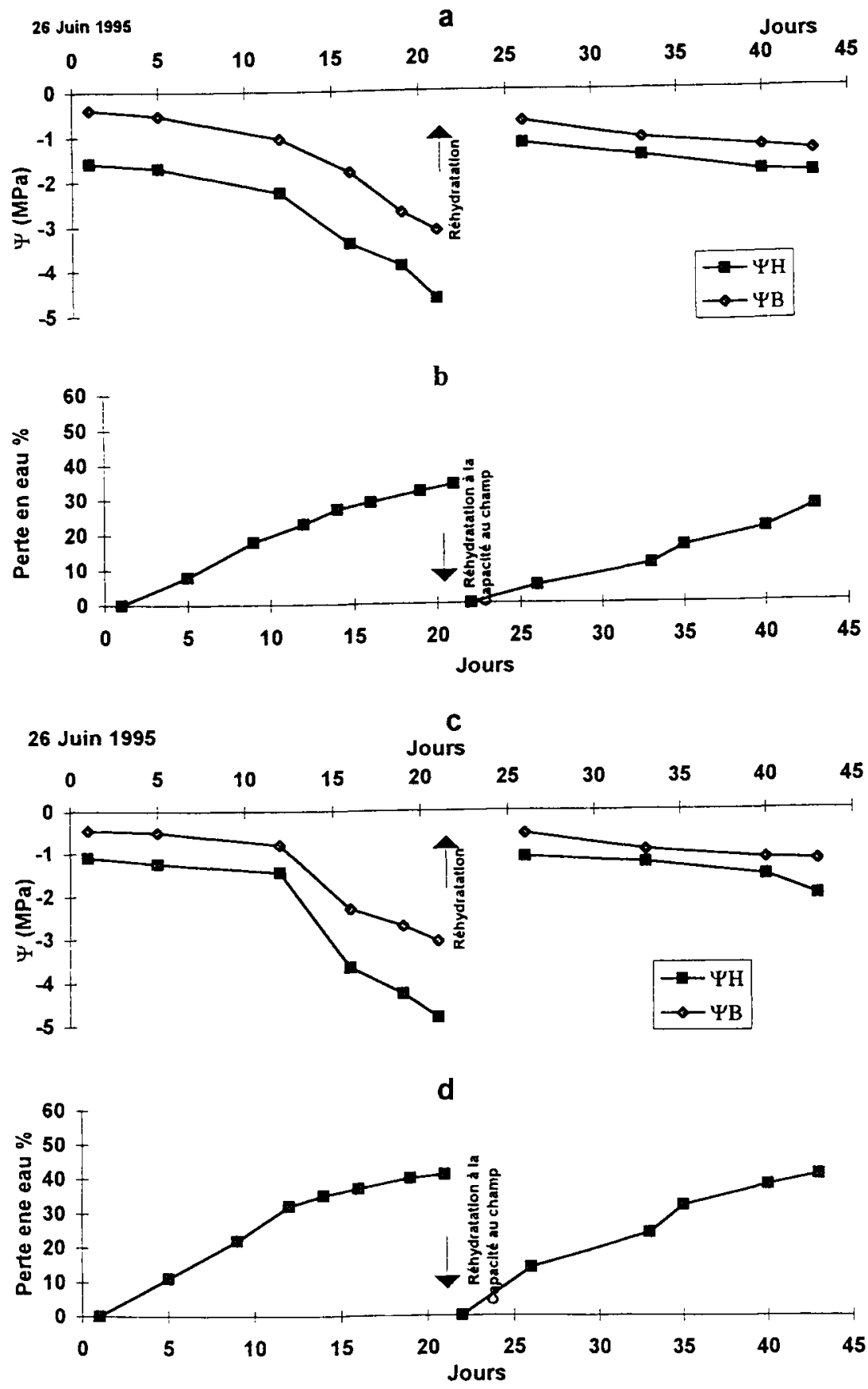

Figure 1b.Variations du potentiel hydrique de base $(\Psi B)$ et du potentiel de milieu de journée $(\Psi H)$, de la perte en eau par rapport à la capacité au champ (Pe) de plants jeunes de chêne liège (a et $b$ ) et de chêne zéen (c et d) pendant deux cycles de sécheresse. 
des valeurs plus basses pour le chêne liège, jeunes plants (CLj) $(-1,6 \mathrm{MPa})$, la valeur la plus élevée est trouvée chez le chêne kermès, jeunes $(\mathrm{CKj})(-0,8)$. À la fin du $1^{\mathrm{er}}$ cycle de dessèchement, les potentiels hydriques de base et minimum les plus bas sont pour CLj de $-3,1$ et $-4,65 \mathrm{MPa}$ respectivement, tandis que les plus élevés sont rencontrés chez le chêne kermès plants âgés (CKa) $-2,45$ et $-3,85 \mathrm{MPa}$ respectivement.

Après la réhydratation et à la fin du $2^{\mathrm{e}}$ cycle de sécheresse, et bien qu'il ait duré autant que le $1^{\text {er }}$ cycle, le potentiel hydrique de base n'a atteint que $-1,4,-1.3$, $-1,2$ et -1,45 MPa pour CLj, CKj, CZj et $\mathrm{CKa}$ respectivement. Il en est de même pour le potentiel hydrique minimum de midi qui varie entre $-1.9 \mathrm{MPa}$ pour $\mathrm{CLj}$ et $\mathrm{CKj},-2,0 \mathrm{MPa}$ pour $\mathrm{CZj}$ et $-2,05 \mathrm{MPa}$ pour CKa.

Les plants âgés de $Q$. coccifera ont connu une baisse progressive de $\Psi_{B}$ jusqu'à la $19^{e}$ journée suivie d'une chute plus rapide. Cette chute se produit dès la $12^{\mathrm{e}}$ journée pour toutes les espèces jeunes particulièrement $Q$. faginea à la fin du $1^{\mathrm{er}}$ cycle (figure $l b c$ ), en dépit d'une surface foliaire supérieure chez les plants âgés $\left(214 \mathrm{~cm}^{2}\right)$ par rapport aux jeunes $\left(163 \mathrm{~cm}^{2}\right)$.

Le deuxième cycle de sécheresse a mis en évidence une réponse physiologique de récupération pour toutes les espèces mais la contrainte hydrique a été moins élevée probablement liée à une adaptation des plantes consécutive au premier cycle de sécheresse.

\subsection{La perte en eau}

Les pertes en eau qui représentent la transpiration cumulée des plantes au cours des deux cycles sont présentés figure lab pour $\mathrm{CKa}, l a d$ pour $\mathrm{CKj}, 1 b b$ pour CLa et Ibd pour CLj.

Les plants âgés de $Q$. coccifera et de même pour $Q$. suber (tableau l) ont pré- senté une perte en eau semblable à la fin du $1^{\text {er }}$ et du $2^{\text {e }}$ cycle de sécheresse qui variait entre 50 et $52 \%$. Chez les jeunes plants et à la fin du $1^{\mathrm{er}}$ cycle, ces valeurs sont de $27 \%$ et $41 \%$ pour CKj et CZj respectivement, ces différences entre jeunes et âgés et entre les différentes espèces jeunes doivent être reliées à l'importance de la surface foliaire qui était en moyenne de $163 \mathrm{~cm}^{2}$ par pot pour $\mathrm{CZj}, 70,3 \mathrm{~cm}^{2}$ pour $\mathrm{CKj}, 214 \mathrm{~cm}^{2}$ et $213 \mathrm{~cm}^{2}$ pour $\mathrm{CKa}$ et CLa respectivement.

\subsection{Variation des paramètres morphologiques}

La biomasse de la tige, de la racine et l'évolution du rapport tige/racine ainsi que la surface foliaire sont présentées (tableau I).

Les analyses statistiques montrent une différence hautement significative au seuil de 0,01 entre espèces pour tous les facteurs étudiés, biomasse racinaire $(\mathrm{R})$, biomasse de la partie aérienne $(\mathrm{T})$, rapport biomasse tige/racine (T/R) et surface foliaire totale $(\mathrm{SF})$, et une différence significative au seuil de 0,05 entre traitements (témoin et stressé).

L'analyse globale des moyennes par le test de Duncan met en évidence une différence significative pour $\mathrm{R}$ et $\mathrm{T}$ entre les stressés et les témoins avec des valeurs inférieures chez les témoins.

Pour T/R et SF, les différences ne sont pas significatives. Par ailleurs, on note une différence significative pour tous les facteurs entre espèces.

On peut noter la croissance supérieure de la partie aérienne des chênes kermès âgés comparés aux chênes lièges, ce qui n'est pas observé pour leur appareil racinaire.

La moyenne générale de l'ensemble des rapports $T / R$ des plants témoins et stressés est plus élevée chez le chêne kermès jeune et âgé avec des valeurs variant 


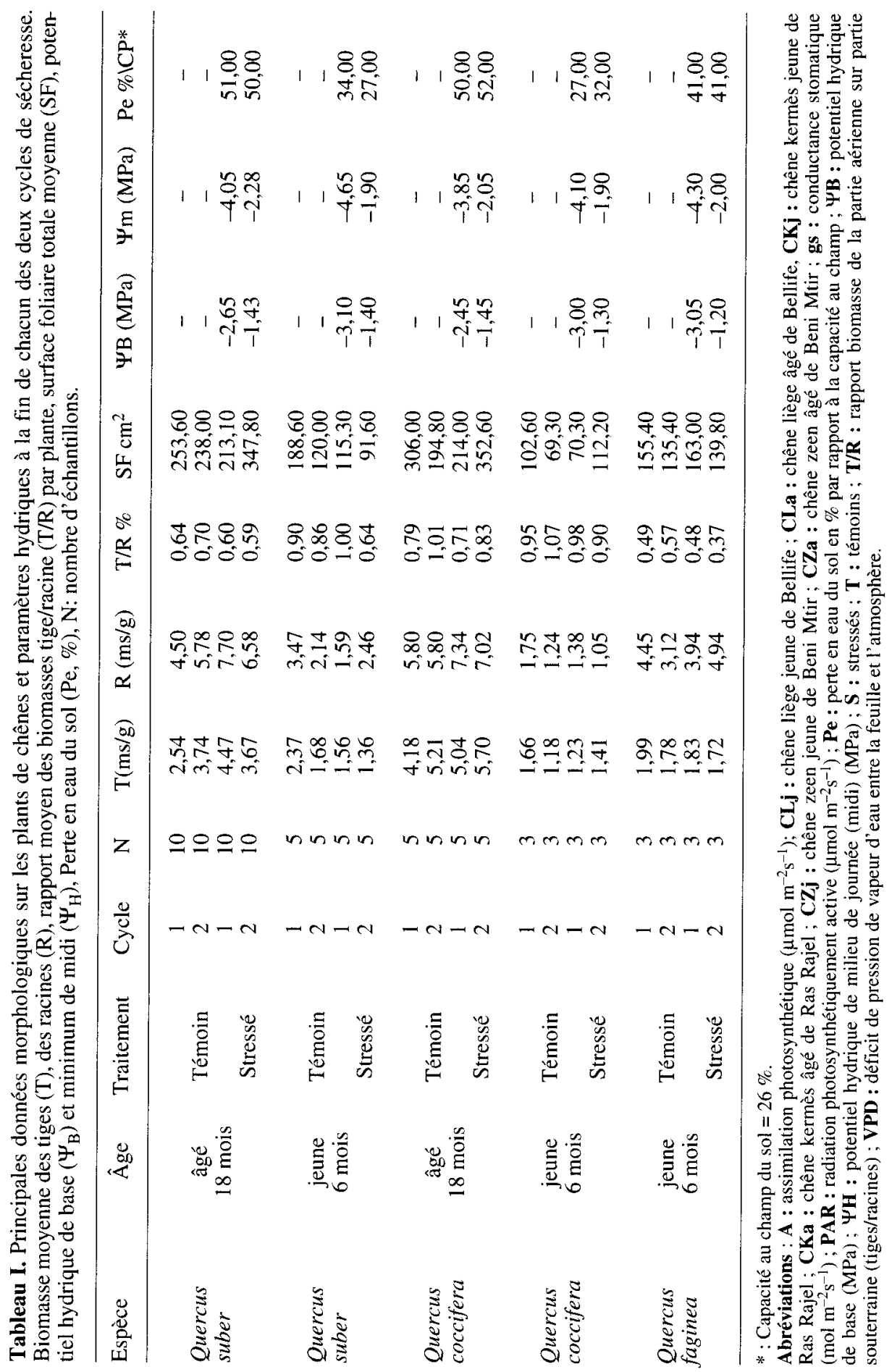


de 0,84 à 0,91 que chez le chêne zéen jeune et le chêne liège âgé avec les valeurs de 0,43 et 0,63 ce qui s'explique par une biomasse racinaire relativement plus importante chez le chêne zeen jeune et une biomasse aérienne plus basse chez le chêne liège âgé.

Les surfaces foliaires totales sont évidemment plus grandes chez les plants âgés mais on peut noter que chez les plants de l'année, le chêne zéen présente une surface foliaire très supérieure à celles des autres espèces.

\subsection{Variation de la conductance stomatique (gs)}

Pendant le deuxième cycle, chez les plants âgés (figure 2), les conductances stomatiques ont augmenté chez les témoins de 0,2 et $0,1 \mathrm{~mol} \mathrm{~m}^{-2} \mathrm{~s}^{-1}$ à 9 heures et midi respectivement pour $Q$. suber et $Q$. coccifera pour atteindre à la fin du $2^{\mathrm{e}}$ cycle 0,60 et $0,45 \mathrm{~mol} \mathrm{~m}^{-2} \mathrm{~s}^{-1}$. Chez les plants stressés, la conductance stomatique à 9 heures est supérieure à celle du milieu de la journée avec cependant une diminution progressive comparable jusqu'à atteindre des valeurs quasi-nulles à la fin du $1^{\text {er }}$ cycle. Après réhydratation, on observe une ouverture stomatique plus importante à 9 heures qu'à midi et plus élevée chez $Q$. suber que $Q$. coccifera avec une gs maximale de l'ordre de 0,25 et 0,35 mol m $\mathrm{m}^{-2} \mathrm{~s}^{-1}$ à 12 heures et 9 heures respectivement pour $Q$. coccifera et de 0,40 et $0,50 \mathrm{~mol} \mathrm{~m}^{-2} \mathrm{~s}^{-1}$ à 12 heures et 9 heures respectivement pour $Q$. suber.

Pour les jeunes plants témoins (figure 3), la conductance stomatique est de l'ordre de 0,10 à $0,20 \mathrm{~mol} \mathrm{~m}^{-2} \mathrm{~s}^{-1}$ à midi et 9 heures respectivement pour toutes les espèces pour atteindre à la fin du $2^{\mathrm{e}}$ cycle 0,40 et $0,60 \mathrm{~mol} \mathrm{~m}^{-2} \mathrm{~s}^{-1}$ et des valeurs de $0,70 \mathrm{~mol} \mathrm{~m}^{-2} \mathrm{~s}^{-1}$ à 9 heures pour $Q$. faginea.

Chez les plants jeunes stressés, la conductance stomatique est plus basse que chez les plants âgés surtout pendant le $1^{\text {er }}$ cycle. Après réhydratation, la récupération de gs initiale n'est que partielle. L'ouverture des stomates des jeunes plants reste limitée malgré un potentiel hydrique de base plus élevé comparé au $1{ }^{\text {er }}$ cycle.

Les conductances stomatiques mesurées sont plus élevées à 9 heures qu'à 12 heures pour toutes les espèces et pendant les deux cycles de sécheresse. Ceci caractérise la fermeture des stomates des chênes en milieu de journée, plus ou moins importante selon l'intensité de la sécheresse et du VPD.

La relation entre gs à 12 heures et le potentiel hydrique de base est montrée figure 4. Une régression polynomiale indique que les relations sont comparables pour $Q$. suber et $Q$. coccifera âgés tandis que chez les plants jeunes, les courbes polynomiales montrent une différence significative pour les différentes espèces. Pour un potentiel hydrique de base de $-2,0 \mathrm{MPa}, Q$. coccifera maintient ses stomates ouverts avec une gs de l'ordre de $0,095 \mathrm{~mol} \mathrm{~m}^{-2} \mathrm{~s}^{-1}$, et pour $Q$. suber de $0,04 \mathrm{~mol} \mathrm{~m}^{-2} \mathrm{~s}^{-1}$, tandis que chez $Q$. faginea, les stomates sont complètement fermés.

\subsection{Variation de l'assimilation nette du $\mathrm{CO}_{2}(\mathrm{~A})$ en fonction du temps}

Pendant le premier cycle de sécheresse, le potentiel hydrique de base ( $\Psi B$ ) a varié de $-0,5 \mathrm{MPa}$ à $-3,0 \mathrm{MPa}$. Les résultats montrent (figure 5) que A est plus élevée à 9 heures qu'à midi pour les deux espèces avec une augmentation importante au cours du temps pour $Q$. suber témoin de 6 à $17 \mu \mathrm{mol} \mathrm{m}^{-2} \mathrm{~s}^{-1}$ et de 4 à $11 \mu \mathrm{mol} \mathrm{m}^{-2} \mathrm{~s}^{-1}$ pour $Q$. coccifera. Chez les stressés, les valeurs ont atteint un minimum de 2 et $3 \mu \mathrm{mol} \mathrm{m} \mathrm{m}^{-2} \mathrm{~s}^{-1}$ pour $Q$. coccifera et $Q$. suber respectivement. Les résultats obtenus montrent une relation étroite entre A et $\Psi B$.

La figure 6 montre la relation entre $\mathrm{A}$ et gs à 9 heures et à 12 heures pour les plants témoins de chêne kermès et chêne liège. 

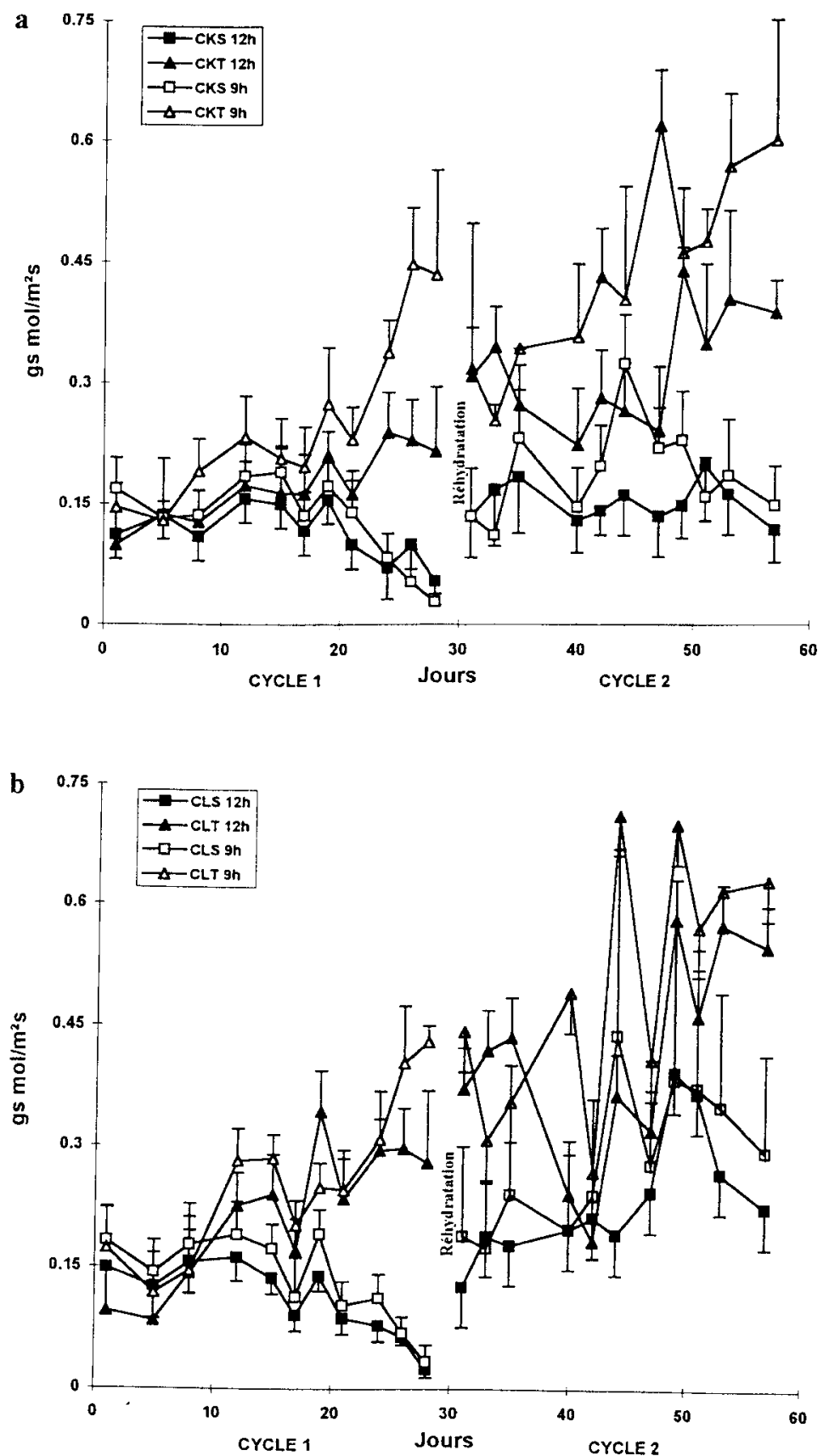

Figure 2. Variations de la conductance stomatique moyenne (gs) au cours de deux cycles de sécheresse chez les plants âgés irrigués et stressés de chêne kermès (CKT et CKS) (a) et de chêne liège (CLT et CLS) (b). 

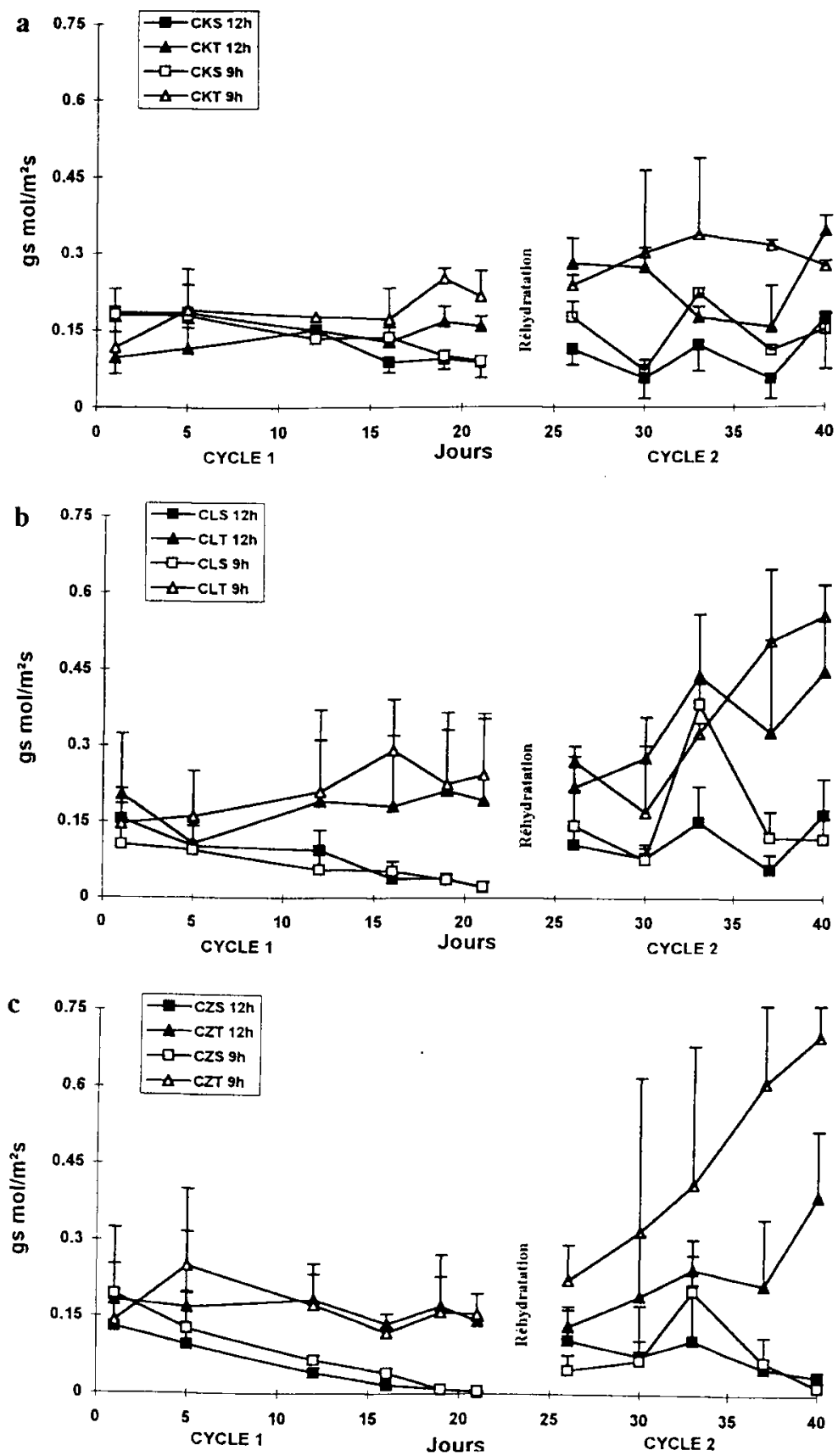

Figure 3. Variations de la conductance stomatique moyenne (gs) au cours de deux cycles de sécheresse chez les plants jeunes irrigués et stressés de chêne kermès (CKT et CKS) (a), de chêne liège (CLT et CLS) (b), de chêne zéen (CZT et CZJ) (c) à 9 heures et 12 heures. 
$\mathbf{a}$

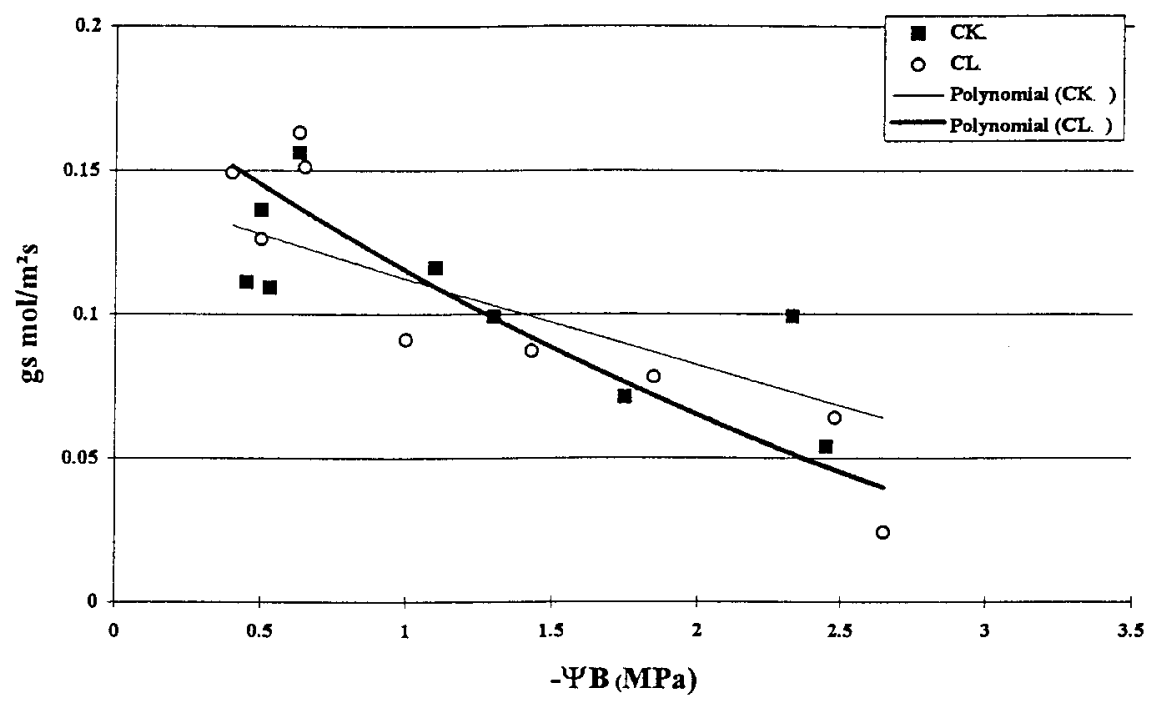

b

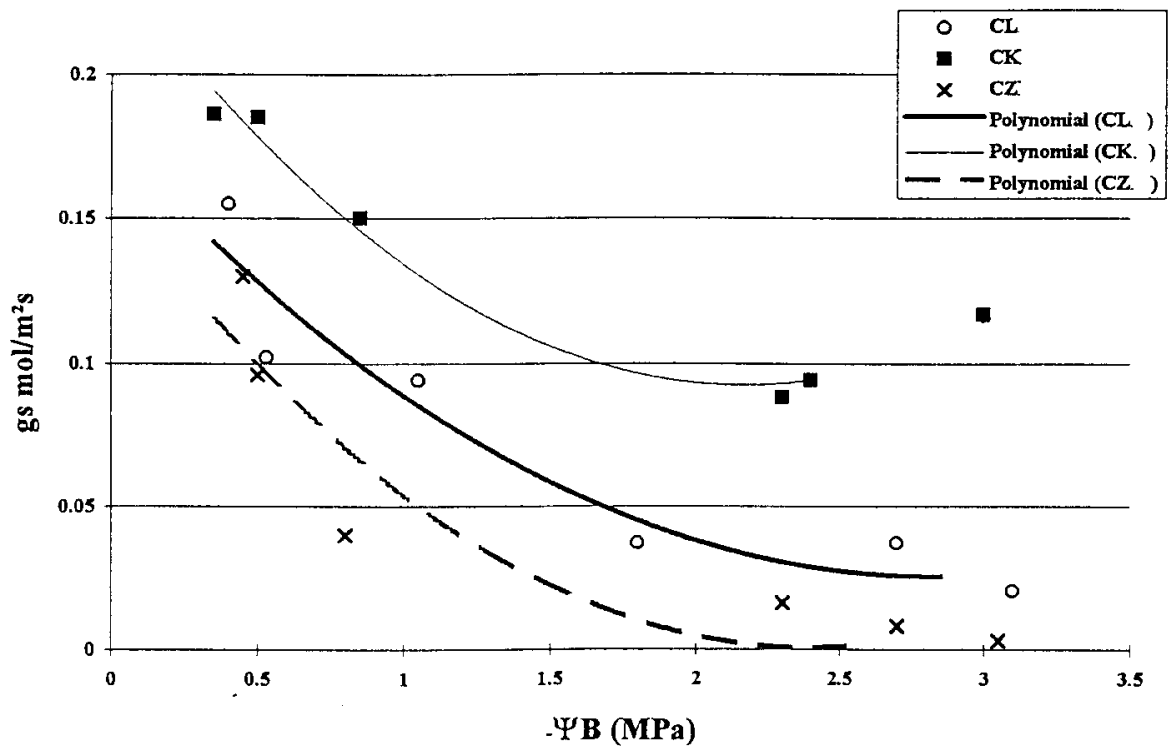

Figure 4. Relation entre la conductance stomatique moyenne à $12 \mathrm{H}$ et le potentiel hydrique de base ( $\Psi B$ ) chez des plants âgés (a) de chêne liège bellife (CL) et de chêne kermès Ras Rajel (CK) et chez des plants jeunes (b) de chêne bellife (CL), de chêne kermès Ras Rajel (CK) et de chêne zéen Mtir (CZ) au cours du premier cycle de sécheresse. 

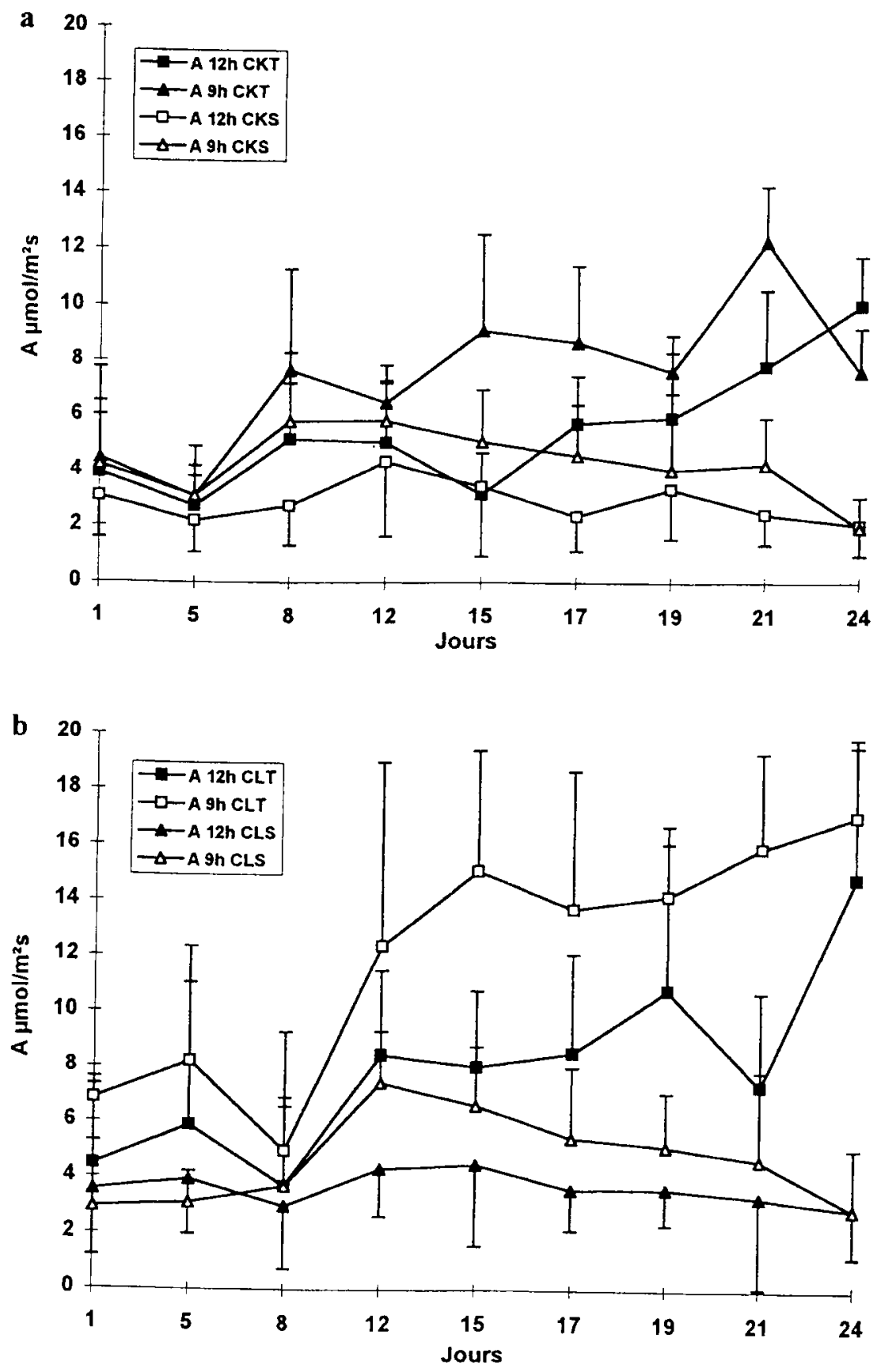

Figure 5. Variations de l'assimilation photosynthétique (A) en fonction du temps chez les plants âgés de chêne kermès Ras Rajel témoins (CKT) et stressés (CKS) (a) et des plants âgés de chêne liège bellife témoins (CLT) ct stressés (CLS) (b) durant le premier cycle de sécheresse. 
Les régressions polynomiales ont été portées pour le chêne liège témoin. Les activités photosynthétiques mesurées à 12 heures sont plus basses que celles mesurées à 9 heures. On peut noter qu'il existe en fonction des jours des différences significatives pour le rayonnement photosynthétiquement actif (PAR).

Les éclairements à 12 heures sont saturants, de l'ordre de $754 \mu \mathrm{mol} \mathrm{m}{ }^{-2} \mathrm{~s}^{-1}$ par rapport à ceux à 9 heures de $432 \mu \mathrm{mol} \mathrm{m} \mathrm{m}^{-2} \mathrm{~s}^{-1}$ et cependant $\mathrm{A}$ à 12 heures est plus faible ; les différences d'intensité d'éclairement ne peuvent donc être la cause des A faibles. En revanche, la température et le VPD plus élevés à 12 heures pourraient expliquer cette réponse.

\section{DISCUSSION ET CONCLUSION}

L'étude de la croissance des organes aériens et souterrains effectuée pour toutes les espèces et durant les deux cycles de sécheresse a complété l'analyse des paramètres physiologiques. Les jeunes plants de chêne possèdent une racine pivot et la contrainte hydrique tend à diminuer le rapport des biomasses aériennes sur racinaires ; cette réponse est fréquemment rencontrée chez les espèces ligneuses ou herbacées et son importance est souvent considérée comme une réponse adaptative à la contrainte hydrique.

Notre étude a montré que sous l'effet du stress $Q$. faginea jeune et $Q$. suber âgé ont un rapport $\mathrm{T} / \mathrm{R}$ inférieur à celui des autres espèces. Les rapports tige/racine de jeunes plants de $Q$. robur, $Q$. petraea, $Q$. rubra

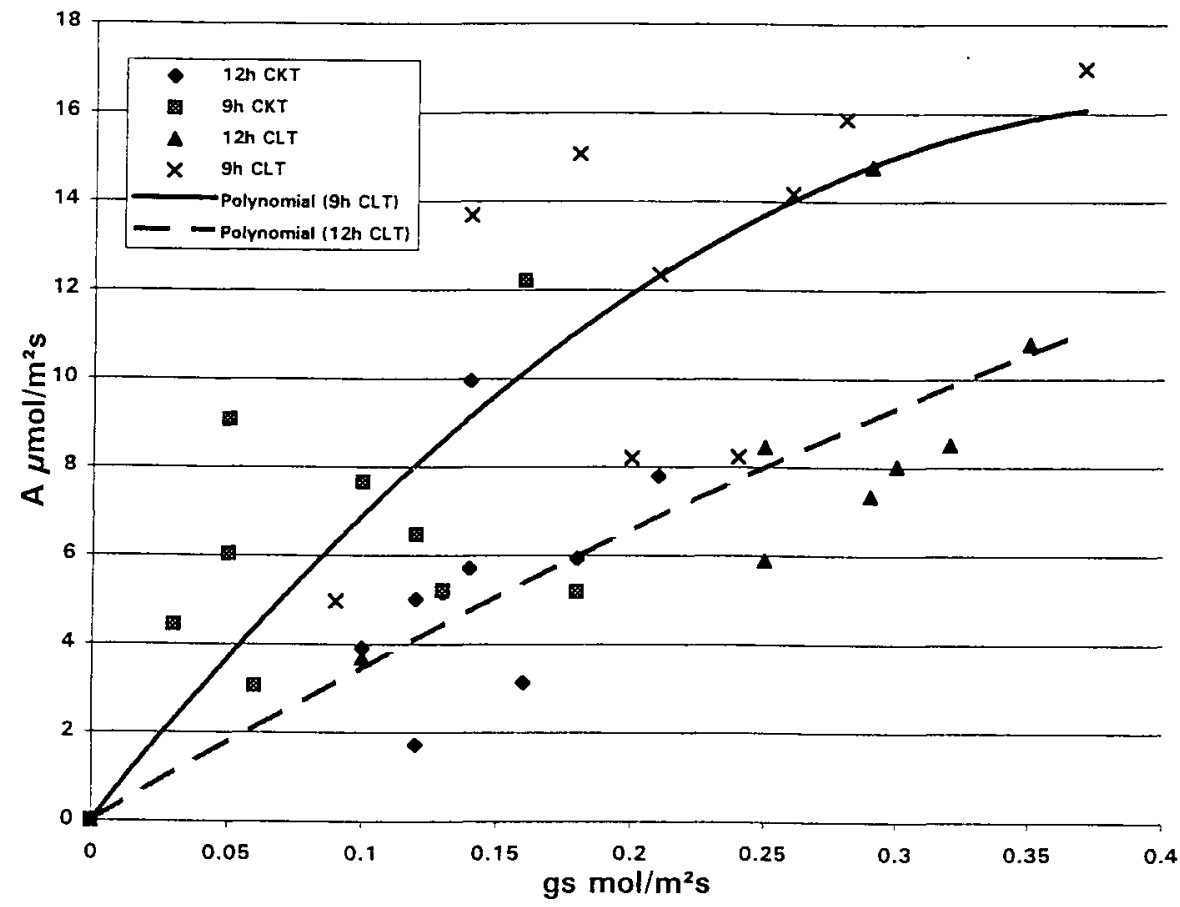

Figure 6. Variations de l'assimilation photosynthétique (A) en fonction de la conductance stomatique (gs) à 9 heures et 12 heures pendant le premier cycle de sécheresse pour des plants âgés de chêne kermès (CKT) et de chêne liège témoins (CLT). 
diminuent selon les espèces de 1,2 à $0,7-0,5$ sous l'effet de la diminution du potentiel hydrique du sol [15].

Champagnat [11] a montré qu'il est difficile d'analyser quantitativement le développement des tiges et des racines des chênes dans des conditions de dessèchement du sol à cause du comportement rythmique de la croissance des tiges qui est affecté dès que le potentiel hydrique de base atteint $-0,1 \mathrm{MPa}[3,43]$. Scuiller [34] a trouvé le même résultat sur des jeunes plants de 3 mois.

D'après Scuiller [34] et Hasnaoui [21], la vitesse d'enracinement après la germination, dans des conditions hydriques limitantes, est liée à l'importance des réserves cotylédonaires.

Le calcul de variance sur les rapports tige/racine montre que les différences entre espèces et entre traitements sont hautement significatives. Canadell et Roda [10], sur $Q$. ilex adultes, montrent également que le rapport des biomasses tige/racine est plus faible en milieu sec. On peut noter que la biomasse racinaire mesurée dans notre expérimentation est identique pour $Q$. coccifera âgé et $Q$. suber âgé. Tenhunen et al. [39] indiquent que $Q$. coccifera et Pistacia lentiscus sont plus adaptés à des conditions arides que $Q$. suber et $A r b u$ tus unedo ce que Rambal [28] explique par une adaptation morphologique des racines leur permettant une meilleure exploitation du sol.

Le potentiel hydrique de base au début du premier cycle de sécheresse est identique pour toutes les espèces quel que soit leur âge. Le potentiel hydrique minimum de midi est plus bas pour les espèces jeunes de $Q$. suber $(-1,6 \mathrm{MPa})$, et plus élevé chez $Q$. coccifera et chez $Q$. faginea jeune. Cette différence de potentiel hydrique $(\Psi H)$ ne peut pas être expliquée par une différence dans les teneurs en eau foliaires et devrait être la conséquence d'un potentiel osmotique plus bas chez $Q$. suber. $\Psi H$ et la teneur relative en eau diminuent plus fortement au cours de la journée chez $Q$. suber comparé à $Q$. ilex selon Salleo et Lo Gullo [33].

À la fin du premier cycle, les jeunes plants ont des potentiels hydriques de base comparables de -3,0 MPa mais il y a une différence de perte en eau par transpiration qui est plus élevée pour $Q$. faginea ; cela pourrait être expliqué par la plus grande surface foliaire et un enracinement plus développé chez $Q$. faginea qui entraîne un rapport tige-racine plus bas en accord avec Acherar et al. [1].

Les plants âgés de $Q$. suber et $Q$. coccifera ont un potentiel hydrique de base et une perte en eau identique de $50 \%$ à la fin du $1^{\text {er }}$ cycle pour une même surface foliaire et un rapport tige/racine qui diffère significativement (tableau I). $Q$ suber présente une masse racinaire plus importante par rapport à la partie aérienne ce qui pourrait augmenter l'intensité de la transpiration révélant une stratégie d'adaptation du type water spenders.

Tenhunen et al. [39] trouvent que le potentiel hydrique de base est toujours plus élevé chez $Q$. coccifera comparé à $Q$. suber en conditions de sécheresse avec une transpiration supérieure de $50 \%$ à celle de $Q$. suber. Cependant, ces auteurs ont expérimenté sur des arbres et dans des conditions d'environnement différentes.

La fermeture des stomates est le principal facteur responsable de la diminution de l'assimilation du $\mathrm{CO}_{2}$ dans des conditions de contrainte hydrique en limitant la diffusion du $\mathrm{CO}_{2}$ vers le chloroplaste [17, 221.

Pendant le $1^{\text {er }}$ cycle de dessèchement, la conductance stomatique décroît quasi linéairement avec le potentiel hydrique de base (figure 4). Les plants âgés de $Q$. coccifera et de $Q$. suber présentent une décroissance comparable également décrite par Acherar et al. [1], Pereira et al. [27], Reich et Hinckley [29] et sur Q. ilex par Sala Serra et al. [32]. La baisse de la 
conductance stomatique est comparable aussi pour les jeunes plants avec cependant une chute précoce pour $Q$. faginea et une fermeture complète des stomates pour un potentiel de base de $-2.5 \mathrm{MPa}$. Cette décroissance plus rapide chez les chênes caducifoliés ( $Q$. faginea et $Q$. afares) a été aussi observée par Acherar et Rambal [2]. Q. suber garde ses stomates ouverts partiellement malgré un faible potentiel hydrique de $-3,0 \mathrm{MPa}$ tandis que chez $Q$. coccifera, pour le même potentiel, la conductance stomatique est de $0,09 \mathrm{~mol} \mathrm{~m}^{-2} \mathrm{~s}^{-1}$. Ce résultat témoigne de la sensibilité supérieure de $Q$. faginea à la contrainte hydrique. La conductance stomatique serait aussi contrôlée par la disponibilité de l'eau dans le sol pour Nerium oleander, une espèce sclérophylle méditerranéenne [18].

Chez les témoins irrigués, on observe pendant la durée de l'expérimentation une augmentation de l'ouverture stomatique accompagnée d'une augmentation de la photosynthèse.

Les conditions d'environnement ne permettent pas d'expliquer cette augmentation. On peut poser I'hypothèse d'une adaptation progressive des feuilles aux conditions climatiques ou encore la possibilité d'une manifestation d'une croissance rythmique [20].

Chez les plants âgés et jeunes de $Q$. coccifera et $Q$. suber, la conductance stomatique est plus élevée à 9 heures qu'à midi pour les plants irrigués et les plants stressés. Il s'agit de la régulation classique de l'ouverture des stomates encore appelée dépression de midi et déjà décrite par de nombreux auteurs sur les espèces méditerranéennes [22, 35]. Tenhunen et al. [36] ont suggéré que cette dépression de midi permet de minimiser la transpiration foliaire et que la fermeture des stomates est déterminée par le VPD. Hinckley et al. [21] l'ont attribué plutôt à l'interaction de certains facteurs notamment le potentiel hydrique foliaire instantané.
Au cours des deux cycles, la conductance stomatique des jeunes plants est restée inférieure à celle des plants âgés à cause d'une part, de leur système racinaire moins développé et, d'autre part, d'un meilleur évitement des jeunes plants pour économiser l'eau. Cette constatation est particulièrement remarquable chez $Q$. fag $i$ nea qui a procédé à une fermeture stomatique rapide, mécanisme physiologique d'évitement de la contrainte hydrique. Pendant le deuxième cycle, gs a continué de progresser chez les plants témoins et âgés avec l'âge des feuilles, ce qui témoigne de leur adaptation progressive aux conditions climatiques. Chez les plants âgés stressés, on observe une augmentation nette de l'ouverture des stomates alors que les stomates des jeunes plants ont toujours une conductance inférieure à celle des plants âgés à la fin du deuxième cycle, particulièrement chez $Q$. faginea.

Cette différence pourrait être liée aux températures moins élevées dans l'expérience plantes âgées. Cependant, on ne peut exclure totalement la possibilité d'une meilleure adaptation dans le cas des plantes âgées.

À la fin du $2^{\mathrm{e}}$ cycle de sécheresse, la mortalité enregistrée chez les plants âgés CK et CL est de l'ordre de 7 et $11 \%$ respectivement pour un potentiel de base $\left(\Psi_{\mathrm{B}}\right)$ final de -1,4 MPA et elle est de 8, 18 et $35 \%$ chez les jeunes plants CK, CL et $\mathrm{CZ}$ respectivement à un $\Psi_{\mathrm{B}}$ de $-1,3 \mathrm{MPA}$.

Hasnaoui (1992) a trouvé sur le terrain des taux de mortalité qui varient entre 40 et $76 \%$ la première année, pour atteindre $100 \%$ la quatrième année sur des jeunes semis plantés artificiellement et des jeunes plants à l'état naturel de $Q$. suber, $Q$. coccifera et $Q$. faginea. Ben Abid et Dhamani [5] ont confirmé ces résultats au Maroc et l'ont attribué essentiellement au manque d'ombrage et à la sécheresse de l'été.

La photosynthèse des plants témoins a atteint, à 9 heures, $18 \mu \mathrm{mol} \mathrm{m} \mathrm{m}^{-2} \mathrm{~s}^{-1}$ pour $Q$. suber et $12 \mu \mathrm{mol} \mathrm{m} \mathrm{m}^{-2} \mathrm{~s}^{-1}$ pour $Q$. coc- 
cifera. Ces valeurs se rapprochent de celles trouvées par Tenhunen et al. [39, 40] pour des arbustes de $Q$. coccifera

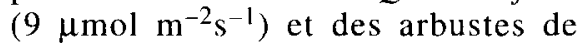

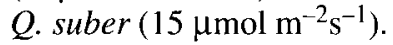

Les conditions de notre expérimentation ont pu influencer l'activité photosynthétique des plants qui sont élevés dans des pots où la disponibilité d'eau est limitée au volume du pot et où la température peut atteindre $30^{\circ} \mathrm{C}$. On note que la photosynthèse est affectée et diminue rapidement au-dessus d'une température de $35^{\circ} \mathrm{C}$ chez les chênes [37].

L'assimilation du $\mathrm{CO}_{2}$ est contrôlée par les mouvements stomatiques surtout dans des conditions de contrainte hydrique. Nos résultats, obtenus pendant un cycle de sécheresse, montrent que la conductance stomatique et l'assimilation photosynthétique sont corrélées et qu'elles présentent toutes deux une dépression de midi. Pour les plants soumis à une contrainte hydrique, A varie entre 6 et $2 \mu \mathrm{mol} \mathrm{m} \mathrm{m}^{-1}$ à 9 heures pour $Q$. suber et $Q$. coccifera avec une diminution de 2 à $3 \mu \mathrm{mol} \mathrm{m} \mathrm{m}^{-2} \mathrm{~s}^{-1}$ pour les valeurs de midi. Cette dépression de midi a été mentionnée par Tenhunen et al. [39] pour $Q$. coccifera. Dreyer et al. [14] et Beyschlag et al. [7] ont montré que la contrainte hydrique fait diminuer $\mathrm{A}$ en parallèle avec gs et que les chênes sont capables de photosynthétiser avec un taux élevé le matin sous des conditions de sécheresse sévère et en fin d'après-midi.

Chez les témoins, les courbes $A=f(g s)$ sont classiques avec une relation linéaire entre $\mathrm{A}$ et gs aux faibles ouvertures stomatiques, puis A présente un plateau de saturation lié à l'existence de la résistance à la diffusion du $\mathrm{CO}_{2}$ dans la couche limite. Mais cette relation diffère pour les valeurs de $\mathrm{A}$ mesurées à 9 heures et à 12 heures. Pour une même gs, A est supérieure à 9 heures.

Les courbes $A=f(g s)$ sont établies généralement en conditions contrôlées de température et pour des éclairements satu- rants. On peut noter que les A les plus faibles pour une gs donnée sont mesurées chez les deux espèces $(Q$. suber et $Q$. coccifera) alors que l'éclairement est le plus élevé. Bien qu'il s'agisse de jeunes plants se développant normalement sous l'ombrage des arbres adultes avec un éclairement limité, il semble peu probable que la baisse de $\mathrm{A}$ soit due à une photoinhibition en présence d'un éclairement solaire direct et malgré la sciaphilie probable des jeunes plants. Roupsard et al. [31] ont montré que la contrainte hydrique diminuait la conductance du mésophylle au $\mathrm{CO}_{2}(\mathrm{gm})$ chez le chêne. Cependant, ces auteurs estiment que les artéfacts connus dans le calcul de $\mathrm{Ci}$ conduisent à la conclusion que la fermeture des stomates serait probablement le facteur limitant de l'approvisionnement en $\mathrm{CO}_{2}$ des chloroplastes en conditions de sécheresse. En revanche, la température des feuilles est plus élevée à midi qu'à 9 heures et nous avons montré, dans des expériences au laboratoire, que les températures supérieures à $30^{\circ} \mathrm{C}$ pouvaient limiter la photosynthèse nette. Il pourrait s'agir d'une action au niveau métabolique par une diminution de l'activité des enzymes de synthèse et/ou une activation du métabolisme dégradatif.

En plus du stress hydrique édaphique, la photosynthèse dépend d'autres paramètres tels que l'intensité lumineuse et la température de la feuille [14]. Ces auteurs indiquent que les chênes, même mésophylles, sont capables de garder l'intégrité de leur appareil photosynthétique sous des conditions de sécheresse sévère ; les feuilles de chênes sont aussi capables de montrer une récupération photosynthétique élevée après réhydratation.

En plus de la régulation stomatique, les modifications structurales des surfaces foliaires et la croissance racinaire permettent aux espèces sclérophylles méditerranéennes de s'adapter aux conditions du milieu. 
Pour conclure, $Q$. faginea, chêne caducifolié, a fermé ses stomates rapidement ce qui confirme son statut de water saver. D'après la classification établie par Dreyer et al. [14], Q. coccifera se comporte comme le plus tolérant à la sécheresse et à un degré moindre, il en est de même pour Q. suber.

Les différences mises en évidence entre jeunes plants de 6 et 18 mois et adultes montrent l'importance du suivi sur le terrain de l'écophysiologie de ces jeunes plants en conditions naturelles pendant les premières années de leur développement. Ces recherches sont nécessaires si l'on veut mieux comprendre les problèmes cruciaux de régénération observés dans les suberaies tunisiennes.

\section{REMERCIEMENTS}

Ce travail a été élaboré dans le cadre du projet de coopération scientifique et technique Franco-Tunisienne sur la régénération du chêne liège. Nous remercions particulièrement Erwin Dreyer, Directeur de recherche au centre de l'Inra, de Nancy à Champenoux pour l'intérêt qu'il a pris à ce travail et pour les nombreuses corrections qu'il a bien voulu lui apporter.

\section{RÉFÉRENCES}

[1] Acherar M., Rambal S., Lepart J., Évolution du potentiel hydrique foliaire et de la conductance stomatique de quatre chênes méditerranéens lors d'une période de dessèchement, Ann. Sci. For. 48 (1991) 561-573.

[2] Acherar M., Rambal S., Comparative water relations of four Mediterranean oak species, Vegetatio 99-100 (1992) 177-184.

[3] Aussenac G., Levy G., Influence du dessèchement du sol sur le comportement hydrique et la croissance du chêne pédonculé et du frêne cultivés en cases de végétation, Ann. Sci. For. 40 (1983) 25l-264.

[4] Ben Abid A., Les écosystèmes forestiers, préforestiers et présteppiques du Maroc, Forêt Méditerranéenne Tome VII, ${ }^{\circ} 1$ (1985) 53-64.

[5] Ben Abid A., Dahmani, La régénération naturelle des forêts marocaines, cas de la suberaie de la Mamora, Rapport de recherche. École nationale Forestière d'Ingénieurs, Salé, Maroc, 1990.
[6] Becker M., Levy G., Le dépérissement du chêne en Forêt de Tronçais. Les causes écologiques, Ann. Sci. For. 39 (1982) 439-444.

17] Beyschlag W., Lange O.L., Tenhunen J.O., Photosynthese und Wasser haushalt der immergrünen mediterraneen Hartlaubpflanze Arbutus unedo L. im Jahresverlaufham Freilandstandort in Portugal. I. Tageslaufe von $\mathrm{CO}_{2}-$ Gaswechsel und Transpiration unter natürlichen Bedingungen Flora 178 (1986) 409-444.

[8] Bohnert H.J., Nelson D.E., Jensen R.G., Adaptations to environmental stresses, Plant Cell 7 (1995) 1099-1111.

[9] Bonneau M., Landmann G., De quoi la forêt est-elle malade? La Recherche 205 (1988) 1543-1553.

[10] Canadell J., Roda F., Root biomass of Quercus ilex in a mountain Mediterranean forest, Can J. For. Res. 21 (1991) 1771-1778.

[11] Champagnat $P$. , Rest and activity in vegetative buds of trees, Ann. Sci. For. 46 (1989) 9-26.

[12] Davis S.D., Patterns in mixed chapparral stands. Differential water status and seedling survival during summer drought, in: Kecley S.C. (Ed.), The California Chaparral. Paradigms Reexamined. Natural History of Los Angeles Country. Sciences serie No34, 1989, pp. 97-105.

[13] Delatour C., Les dépérissements du chêne en Europe, Rev. For. Fr. 35 (1983) 265-282.

[14] Dreyer E., Granier A., Bréda N., Cochard H., Epron D., Aussenac G., Oak trees under drought constraints: ecophysiological aspects. Proceedings of an International Congress, Universita Degli Studi, Bari, Italy, 1993, p. 322.

[15] Durand P., Gelpe J., Lemoine B., Riom J., Timbal J., Le dépérissement du chêne pédonculé dans les Pyrénées atlantiques, Rev. For. Fr. 35 (1983) 357-368.

[16] Emberger L., La végétation de la région méditerranéenne. Essai d'une classification des groupements végétaux, Rev. Gen. Bot. 42 (1930) 641-62.

[17] Epron D., Dreyer E., Photosynthesis of oak leaves under water stress: maintenance of high photochemical efficiency of photosystem II and occurrence of nonuniform $\mathrm{CO}_{2}$ assimilation. Tree Physiol. 13 (1993) 107-117.

[18] Gollan T., Turner N.C., Schulze E.D., The response of stomata and leaf gas exchange to vapor pressure deficits and soil water content. III, in: The Sclerophyllous Woody Species Nerium oleander, Oecologia 65 (1985) 356-362.

[19] Hasnaoui B., Étude structurale des trouées de régénération naturelle en forêt humide de plaines d'Alsace. Thèse, U.L.P. Strasbourg, $1979,137 \mathrm{p}$.

[20] Hasnaoui B., Chênaies du Nord de la Tunisie. Ecologie et Régénération. Thèse, Université De Provence-Aix-Marseille I, 1992, 202 p. 
[21] Hinckley T.M., Duhme F., Hinckley A.R.,

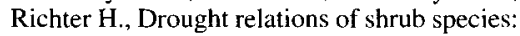
assessment of the mechanisms of drought resistance, Oecologia 59 (1983) 344-350.

[22] Larcher W., De Moraes J.A.P.V., Bauer H., Adaptive responses of leaf water potential, $\mathrm{CO}_{2}$-gas exchange and water use efficiency of Olea europea during drying and rewatering, in: Margaris N., Mooney H. (Eds.), Components of Productivity of Mediterranean Climate Regions. Basic and Applied Aspects, Junk, The Hague, 1981, pp. 77-84.

[23] LePoutre B., Régénération artificielle du chêne liège et équilibre climatique de la subéraie de la forêt de Mamora, Ann. Res. For. Maroc, Tome IX (1965) 1-1 49.

[24] Macaire A., Le dépérissement du chêne pédonculé en forêt communale d'Amance (Aube), Rev. For. Fr. 36 (1984) 201-205.

[25] Marion T., Les repeuplements artificiels en chêne-liège dans la forêt de la Mamora, Ann. Rech. Forest. Maroc (1955) 54-55.

[26] Natividade J.V., Subericultura. Ministra da Economia, Direç̧ao General dos servicos Florestais e Aquicolas Lisboa, 1950.

[27] Pereira J.S., Tenhunen J.D., Lange O.L., Stomatal control of photosynthesis of Eucalyptus globulus labil trees under field conditions in Portugal, J. Exp. Bot. 38 (1987) 1678-1688.

[28] Rambal S., Water balance and pattern of root water uptake by a Quercus coccifera L. evergreen shrub, Oecologia 62 (1984) 18-25.

[29] Reich P.B., Hinckley T.M., Influence of predawn water potential and soil-to-leaf hydraulic conductance on maximum daily leaf diffusive conductance in two oak species, Funct. Ecol. 3 (1989) 719-726.

[30] Rhizopoulos S., Mitrakos K., Water relations of evergreen sclerophylls. I. Seasonal changes in the water relations of eleven species from the same environment, Ann. Bot. 65 (1990) 171-178.

[31] Roupsard O., Gross P., Dreyer E., Limitation of photosynthetic activity by $\mathrm{CO}_{2}$ availability in the chloroplasts of oak leaves from different species and during drought, Ann. Sci. For. 53 (1996) 243-254.

[32] Sala Serra A., Buriel J.A., Tenhunen J.D., Spatial and temporal controls on transpiration within a watershed dominated by Quercus ilex, in: International workshop. Quercus ilex $\mathbf{L}$. Ecosystems: Function and Management, Montpellier and Barcelona (1990) 89-92.

[33] Salleo S., Lo Gullo M.A., Sclerophylly and plant water relations in three Mediterranean Quercus species, Ann. Bot. 65 (1990) 269-270.

[34] Scuiller 1., Exploitation de la variabilité des comportements écophysiologiques de semis de chênes blanes européens soumis à la sécheresse Thèse Université de Nancy I, 1990, 103p.
[35] Tenhunen J.D., Lange O.L., Pereira J.S., Losch R., Catarino F., Midday stomatal closure in Arbutus unedo leaves: measurements with a steady state porometer in the Portuguese evergreen shrub, in: Mogaris N.S., Mooney H.A. (Eds.), Components of Productivity of Mediterranean Climate regions. Basic and Applied Aspects, The Hague, Junk, 1981, pp. 61-69.

[36] Tenhunen J.D., Lange O.L., Braun M., Midday stomatal closure in Mediterranean-type Sclerophylls under simulated habitat conditions in an environmental chamber. II. Effect of the complex of leaf temperature and in humidity on gas exchange of Arbutus unedo and Quercus ilex, Oecologia 50 (1981) 05-11.

[37] Tenhunen J.D., Lange O.L., Gebel J., Beyschlag W., Weber J.A., Changes in photosynthetic capacity, carboxylation efficiency, and $\mathrm{CO}_{2}$ compensation point associated with midday stomatal closure and midday depression of net $\mathrm{CO}_{2}$ exchange of leaves of Quercus suber, Planta 162 (1984 a) 193-203.

[38] Tenhunen J.D., Meister H.P., Cahamell M.M., Lange O.L., Environmental constraints on productivity of the Mediterranean sclerophyll shrub Quercus coccifera. Proceedings of INTECOL workshop. Rates of Natural Primary Production and Agricultural Production. Zaragoza, Spain, Options méditerranéennes 84 (1984) 33-53.

[39] Tenhunen J.D., Lange O.L., Harley P.C., Beyschlag W., Meyer A., Limitations due to water stress on leaf net photosynthesis of Quercus coccifera in the Portuguese evergreen shrub, Oecologia 67 (1985) 23-30.

[40] Tenhunen J.D., Beyschlag W., Lange O.L., Harley P.C., Changes during summer drought in leaf $\mathrm{CO}_{2}$ uptake rates of macchia shrubs growing in Portugal: limitations due to photosynthetic capacity, carboxylation efficiency, and stomatal conductance, in: Tenhunen J.D. et al. (Eds.), Plant Response to Stress. Functional Analysis in Mediterranean Ecosystems, NATO ASI, Series, vol. 615, Springer Verlag, Berlin, 1987, pp. 305-327.

141] Vannini A., Susceptibility of Quercus cerris to Hypoxylon mediterraneum at different levels of water stress, in: Siwecki R., Liese W. (Eds.), Proceedings International Symposium: Oak Decline in Europe, Kornik, Pologne, 1990, pp. I65-172.

[42] Vignes D., Les échanges gazeux et leur régulation chez deux espèces de chênes à feuilles persistantes (Quercus ilex et Quercus suber), Bull. Soc. Bot. Fr. 135, Actual bot. 1 (1988) 99-108.

[43] Vivin P., Aussenac G., Lévy A., Differences in drought resistance among three deciduous oak species grown in large boxes, Ann. Sci. For. 50 (1993) 221-233. 